\title{
تعليم اللغة العربية في المعهد الإسلامي بفونوروغو
}

Moh. Munir

IAIN Ponorogo

moh.munir@iainponorogo.ac.id

\begin{tabular}{|l|l|l|}
\hline Received: November 17th 2019 & Revised: December $4^{\text {th }} 2019$ & Accepted: December $30^{\text {th }} 2019$
\end{tabular}

Abstract: Learning Arabic in Indonesia has experienced the ups and downs along with the dynamics of education in Islamic educational institutions. It gives good attention in Arabic teaching is Islamic boarding school. The Islamic boarding school students have not been able to master language skills well, like Arrisalah concerns with speaking skill and Al amin Hudatul Muna 1 concerns with reading skill. This research focused on three aspects: the materials, the instrutions process and the kinds of languages activities in Arabic teaching in modern and salaf Islamic boarding school for class two. It is kind of qualitative study design to describe the results and collecting the data.The results of this study are: (1) the Arabic teaching materials in modern Islamic boarding school consist of 7 subjects, and salaf consist of 5 subjects. (2) the instructional method in modern Islamic boarding school are used grammar translation method, direct method, audio lingual method, reading method and inductive method. The instructional method in salaf islamic boarding school are used grammar translation method and deductive method. (3) the kinds of language activities in modern Islamic boarding school devided into two kinds namely language activities inside the class and language activities outside the class.

Keywords: islamic boarding school, teaching method, language activities

$$
\begin{aligned}
& \text { إنّ اللغة العربية من أهم وسائل اتصال الإنسان بغيره، والإنسان في حاجة إلى هذا } \\
& \text { الاتصال اللغوى وتدعيمه، كي يعيش في حب وسلام.والعالم اليوم بحاجة إلى أن يكتسب } \\
& \text { الإنسان فيه أكثر من لغة، وهذه الحاجة تتبع من ترابط المصالح الإجتماعية والسياسية }
\end{aligned}
$$


والاقتصادية لجميع الدول وتشابكها في عصرنا الحاضر. 1 ومن إحدى اللغات في العالم هي

اللغة العربية. وصلت اللغة العربية في إندونيسيا منذ دخول الإسلام إلى هذه الدولة في القرن الأول الهجري الموافق بالقرن السابع الميلادي، استنادا إلى السجلات الصينية. وأول منطقة دخلها هي منطقة أتسية، ومنها انتشر الإسلام إلى كل الولايات الموجودة في جنوب

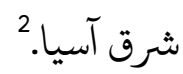

إن تعليم اللغة العربية في إندونيسيا يمر بمراحل طويلة منذ دخول الإسلام إلى هذا اليوم، ذلك في المؤسسات التربوية سواء أكانت حكومية أم أهلية ت تدرس اللغة العربية كما تدرس المواد الأخرى. وتدرس اللغة العربية في المؤسسات التربوية الإسلامية لجميع الطلاب. نجد في بعض المدارس والمعاهد التي أنشئت في السنوات الأولى بعد الاستقلال، فهذه المدارس والمعاهد تقوم بعملية التعليم بناء على أن اللغة محتاجة في قراءة النصوص العربية لفهم مضمونها فحسب. فلا حاجة عند ذاك إلى أن تستخدماللغة العربية لغيرها مثل المحاورة والمكلمة وآلة الاتصال بين الهشر.4

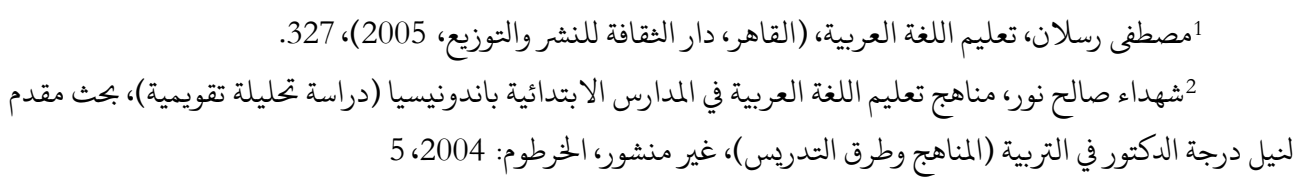

${ }^{3}$ Abdul Hamid, Uril Bahruddin, Bisri musthofa, Pembelajaran Bahasa Arab Pendekatan, Metode, Strategi, Materi dan Media, (Malang: UIN Malang Press, 2008), 158

4أحمد صالح الدين، تعليم اللغة العربية في المعهد السلفي والمعهد العصري (دراسة مقارنة وتقويمية في تعليم اللغة العربية بين معهد ليربويو السلفي وبقديري والأمين العصري بسومنب)، بحث مقدم لنيل درجة الماجستير في التربية، 2007، 
ومن المؤسسات التعليمية التي كانت عندها اهتمام كبير بتعليم اللغة العربية هي

المعهد. ومن المعهد الذي عنده اهتمام كبير بمفهوم ه السلفي هو المعهد الإسلامي السلفي للبنات "الأمين" هداة المنى 1 بجينس. يعلّم المدرس الطلاب بالطريقة التقليدية، حيث يقرأ المعلم كل الكمات ويترجمها إلى اللغة الجاوية ثم يكتب الطلاب المعنى من كلام المعلم. ومن المعهد الذي عنده اهتمام كبير بمفهوم العصري كمعد الرسالة العصريبسلاهونج. يعلّمالمدرس الطلاب بالطريقة الحديثة. وهذا الوضع يتوقع أن يكون ناتجا من اختلاف مواد التعليم والطريقة التعليمية والنشاطات لمعمولة في المعهدين. فهذه المقالة محاولة متواضعة من الباحث في كشف ووصف الظواهر من الواقعية في تعليم اللغة العربية في المهعد الإسلامي من حيث المواد الدراسية وطرق التعليم وتطبيق النشاطات اللغوية.

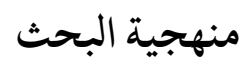

تسر هذهالمقالة على سبيل البحث الكيفي ( Qualitative Research) والبحث الكيفي هو إجراءة البحث التى تنتج البيانات الوصفية ( Descriptive Data أصوات المكتوبة أو من ألسنة الأشخاص والطبائع المتأملة. 5صادر البيانات هي المصادر التي 


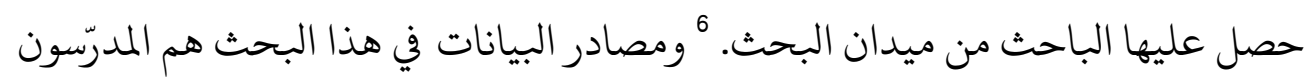
والطلاب في معهد الرسالة بسلاهونج والمعهد الإسلاي السلفي للبنات "الأمين" هداة المنى 1

الأدوات المستخدمة في جمع البيانات هي المقابلة و الملاحظة والوثيقة. استخدمت

المقابلةلأخذ البيانات المتعلّقة بأهداف تعليم اللغة العربية وطرق التدريس

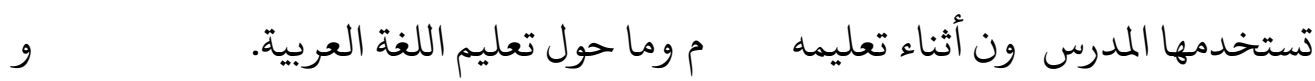

استخدمثلملاحظة لتحصيل البيانات عن المواقع الجغرافي وواقع تعليم اللغة العربية

وبالذات ما يتعلق بتطبيق النشاطات اللغوية في المعهد الرسالة بسلاهج والمعهد الإسلامي

السلفي للبنات"الأمين" هداة المنى 1 بجينس. بينما الوثيقة استخدمتلجمع البيانات عن التراث التحريرى، والكتب وغيرها التي لها صلة بالبحث.

وفي تحليل البيانات استخدم الباحث أسلوبتحليل البيانات الوصفيةالكيفية على

طراز ميلس (Miles) وهوبيرمان (Huberman).

نتائج البحث ومناقشتها

الهواد الدراسية في تعليم اللغة العربية في المعهد الإسلامي بفونوروغو

${ }^{6}$ Suharsimi Arikunto, Prosedur Penelitian Suatu Pendekatan Praktek, (Jakarta PT. Rineka Cipta, 2002), 115 246

${ }^{7}$ Sugiyono, Metode Penelitian Kuantitatif Kualitatif R\&D, (Bandung: Alfabeta, 2011),

Program Studi Pendidikan Bahasa Arab Universitas Yudharta Pasuruan https://jurnal.yudharta.ac.id/v2/index.php/studi-arab 
إن المراد بالمواد الدراسية هو تلك المواد التي تدرس للتلميذ داخل الفصل في مجالات من المعرفة المختلفة، يأخذها التلميذ ليصل بها إلى مستوى ما، أو ليتخرج من

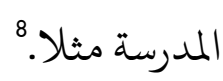

ثم إن المواد الدراسية قد نظمت على واحدة من الأشكال الثلاثة، هي ما يسىى

بالمواد المنفصلة، والمواد المترابطة، والمواد في المجالات الواسعة. تكون المواد الدراسية موادا منفصلة ما كانت المواد تقوم بنفسها حيث لها منهج منفصل عن غيره ولها حصتها ونفذ له تقويم خاص، بينما المواد المترابطة هي المواد تشبه المواد المنفصلة غير أن لهاصلة قوية بغيرها من المواد إما عرضية وإما طولية. تكون المواد الدراسية في المجالات الواسعة عن طريق الدمج فيه بين مادتين أو أكثر التي تكون الصلة بينها وثيقة. و والقصد هنا إدماج الحقائق والمبادئ الضرورية لفهم موضوع معين دون نظر للحدود الفاصلة بين المواد الدراسية، كما في الدراسة الأحياء، ودراسة العلوم العامة، والرياضيات العامة، وكذلك

$$
\text { الاجتماعيات التي تشمل علوم التاريخ والجغرافية والمجتمع. }
$$

نظرا إلى تاريخ المعهد سابقا، أن الدروس الأساسيى في المعهد تدور حول قراءة

القران والصلاة (تعليم الصلاة) وقراءة الكتب. ثم فصلت بعد ذلك إلى ثلاثة أقسام: 1)

$$
\begin{aligned}
& \text { 8أوريل بحر الدين، تطوير منهج تعليم اللغة العربية وتطبيقه على مهارة الكتابة، (مالانج: مطبعة الجامعة الإسلامية } \\
& \text { الحكومية، 2010)، } 32
\end{aligned}
$$

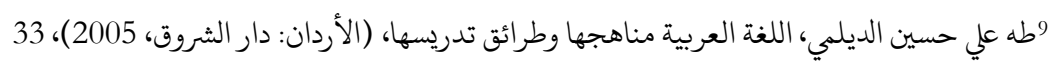

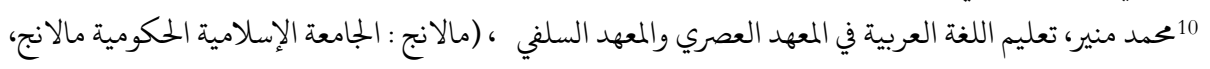

$$
\begin{aligned}
& \text { رسالة دكتوراه، غير منشورة، 2014)، } 56
\end{aligned}
$$

Program Studi Pendidikan Bahasa Arab Universitas Yudharta Pasuruan https://jurnal.yudharta.ac.id/v2/index.php/studi-arab 
العقيدة أو التوحيد، 2) الشريعة أو الفقه، 3) التصوف أو الأخلاق ."1أما العلوم الأخرى فتهكون مساعدة لها ومكملة أو متممة. أما اللغة العربية فتدرس على نهج نظرية الفروع التي تتكون من درس النحو والمحادثة والكلام والإنشاء والمطالعة والترجمة وغير ذلك. أما المواد الدراسية في تعليم اللغة العربية في معهد الرسالة والمعهد الإسلاي

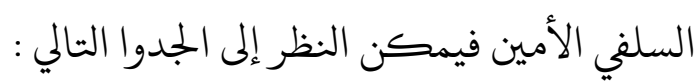

\begin{tabular}{|c|c|c|}
\hline المعهد الإسلامي السلفي & معهد الرسالة العصري & المادة \\
\hline مجادة هو علم النحوجاون الذي ألفه & الكتل المتاب المدرسى المستخدم في & النحو \\
\hline أُعا ألفه إمام شيخ شريف المادة هو كتاب الصرف الدين المستخدم في & تعليم مادة الصرف هو كتاب المدرسي المستخدم في & الصرف \\
\hline
\end{tabular}

$$
\text { 11 وثيقة تاريخ المعهد، الوثيقة 1، فونوروغو، التاريخ } 30 \text { مارس } 2019
$$




\begin{tabular}{|c|c|c|}
\hline من المعهد الإسلامي السلفي الفلاح & المدرسين بكلية المعلمين & \\
\hline - & 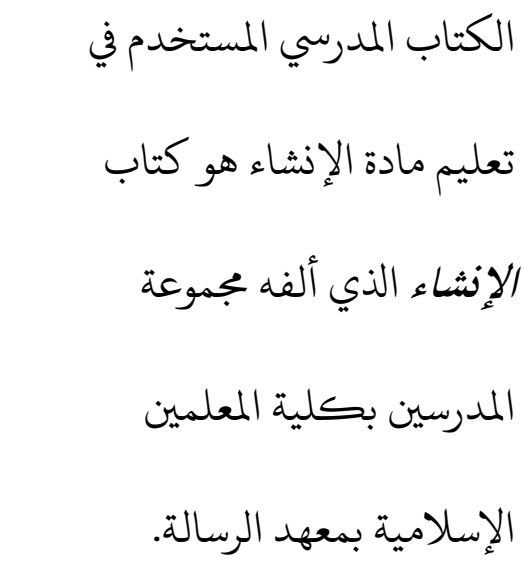 & الإنشاء \\
\hline - & 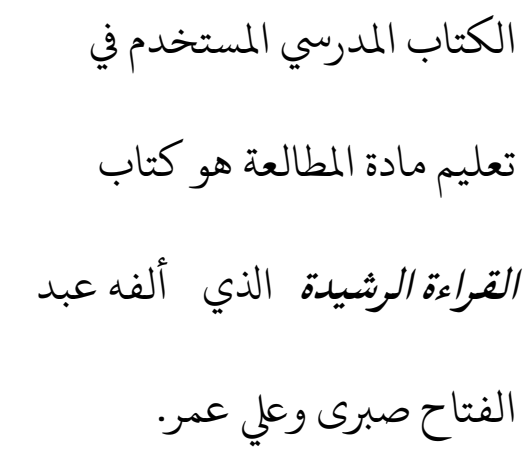 & المطالعة \\
\hline- & الكتاب المدرسي المستخدم في & المحفوظات \\
\hline
\end{tabular}




\begin{tabular}{|c|c|c|}
\hline & الرسالة. & \\
\hline- & 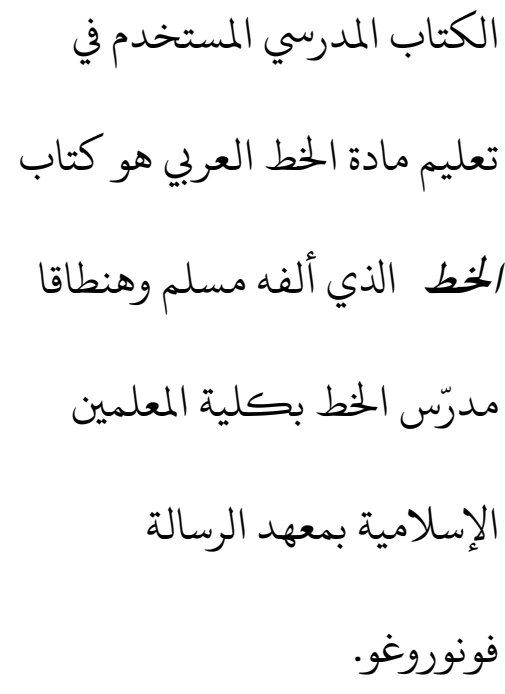 & الخط \\
\hline- & 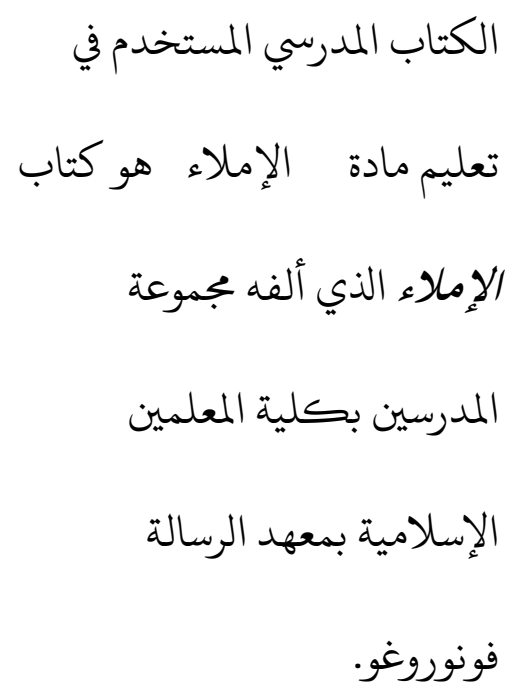 & الإملاء \\
\hline الكادة جرومية هو كتاب المدري المبتخدم في تعليم & - & جرومية \\
\hline
\end{tabular}




\begin{tabular}{|c|c|c|}
\hline فحمد بن داوود الصنهاج من مندينة & & \\
\hline المادة الذمريطي هو كتاب المدرسي المستخدم في تعليم & - & عمريطي \\
\hline الكتاب المدرسي المستخدم في تعليم & - & ألفية \\
\hline
\end{tabular}

انطلاقا م ن الجدول السابق رأى الباحث نوعين مخ لـفين من المواد الدراسية.

فالنوع الأول في معهد الرسالة حيث تجري عملية التعليم بطريقة معاصرة ومواد مختلفة من اللغة العربية المناسبة لأهداف تعليم اللغة العربية. والنوع الثاني في المعهد الإسلاي السلفي الأمينحيث تجي عملية التعليم بطريقة تقليدية وموادمتخصصة منفصلة ومناسبة لأهداف تعليم اللغة العربية. 
يتميز المعهد العصري في تعليم اللغة العربية بإتقان طلابه ممارسة الكلام العربي بل وقد مارسوا المهارات اللغوية الأخرى. تقسم الحصص الدراسية إلى جيع المواد الدراسية المتعلقة بالمهارات الأربعة. فالط لابتعلموا اللغة العربية لوظائفها المختلفة، للاتصال بين الأفراد ولفهم الكتب العربية وفهم الحوار وقواعد اللغة ويتدربون على الكتابة والاستماع

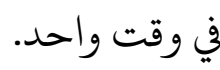

قد يمارس طلاب معهد الرسالة العصري القدرة على الاتصال والتكلم باللغة العربية، حيث يطبق هذا المعهد بيئة لغوية عن طريق إيجاب الطلاب على محارسة الكلام والاتصال بالكلام العربي بعضهم بعضا ـ فهذا النظام يحتاج إلى أن يّـرب الطلاب على الكلمات والعبارات التي تشجعهمعلى التكلم باللغة العربية. فيظظم المعهد موادا دراسية تسهل وتساعد الطلابتطبيق اللغة العربية في يومياتهم. فعن هذا الغرض، لا تتركز المواد الدراسية المطبقة في المعهد العصري على القواعد اللغوية، بل توظف هذه القواعد في ممارسة كل المهارات اللغوية المقصودة. فمن هنا، نجد أنواعا من المواد الدراسية في اللغة العربية، مثل المطالعة والإنشاء وتزويد المفردات، ولا تترك م ادة تتعلق بقواعد اللغةوهي النحو والصرف ، بل نركلن المهدوضع اللغة العربية موضعها الحقيقي حيث يستفيد الطلاب اللغة العربية من جميع المهارات وليس من مهارة واحدة. 
إن هذه المواد الدراسية نظمت تنظيما منطقيا على أساس المواد المترابطة حيث أن

لكل مادة علاقة قوية بغيرها. هذه العلاقة القوية قد تكون علاقة عرضية وقد تكون

طولية.12

\section{طريقة تعليم اللغة العربية في المعهد الإسلامي بفونوروغو}

طريقة التعليم هي الأسلوب الذي يقدم به المدرس المعلومات والحقائق للطلبة، أو

هي الأسلوب الذي ينظم به المدرس الموافق والخبرات التي يريد أن يضع طلبته فيها حتى تتحقق لديهم الأهداف المطلوبة.13

يهدف تعليم اللغة العربية في معهد الرسالة إلى تنمية قدرة الطلاب في المهارات

اللغوية الأربعة هي الكلام والإستماع والقراءة والكتابة. طبق المعهد الرسالة في تعليم اللغة العربية الطرق الحديثة كال طريقة المباشرة وطريقة السمعية الشفهية وطريقة القراءة، والطريقة الإستقرائية في تعليم النحو.

وأما في المعهد السلفي يسير التربية بالطريقة التقليدية يعنى: التقديم (sorogan) و

التوقيت (weton). طريقة التقديم هي الطريقة التي تعطي للطلاب فرصة لفتح الكتاب المعين أو القرآن ويقدمون قراءتهمإلى المدرس واجدا فواحداً ، بينماطريقة التوقيت هي

$$
12 \text { طه علي حسين الديلي، اللغة العربية مناهجها وطرائق تدريسها، } 22
$$

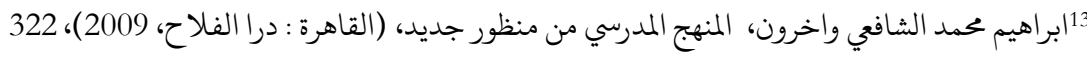

Program Studi Pendidikan Bahasa Arab Universitas Yudharta Pasuruan https://jurnal.yudharta.ac.id/v2/index.php/studi-arab 
الطريقة التي تعطي للمدرس فرصة قراءة الكتاب ومعاني المقروء أمام الطلابفي حين أن الطلاب يستمعون إليه ويكتبون معاني الألفاظ الغامضة عندهم وأحيانا يستعمل المدرس طريقة القواعد والترجمة والطريقة القياسية في تعليم النحو لتسهيل فهم الطلاب. يسير هذا النعليم كل ليلة وصباح بعد صلاة الصبح. فلمزيد من البيان السايق، يمكن النظر إلى الجدول التالي :

\begin{tabular}{|c|c|c|c|}
\hline \multirow[t]{2}{*}{ 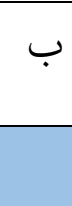 } & \multirow[t]{2}{*}{ i } & طريقة التعليم & 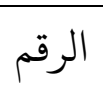 \\
\hline & & استخدام طريقة النحو والترجة & 1 \\
\hline & & استخدام طريقة السمعية الشفهية & 2 \\
\hline & & استخدام طريقة المباشرة & 3 \\
\hline & & استخدام طريقة القراءة & 4 \\
\hline & & استخدام طريقة الإستقرائية في تعليم النحو & 5 \\
\hline & & استخدام طريقة القياسية في تعليم النحو & 6 \\
\hline & & 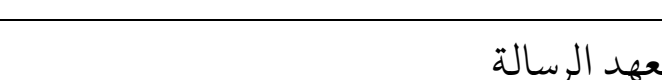 & \\
\hline
\end{tabular}

$$
\text { ب :المعهد الإسلاي الأمين }
$$

يجب أن تكون طرق تعليم اللغة العربية المستخدمة مناسبة للمواد الدراسية. فمن المفروض أن تكون الطرق المستخدمة تساعد الط لاب في فهم المواد الدراسية بسهولة، وهذا يتبني على نوع المادة المدروسة. من هنا نرى في معهد الرسالة أنواعا من الطرق 
المستخدمة وهي الطريقة القواعد والترجمةوالطريقة المباشرة والطريقة السمعية والشفوية وطريقة القراءة.

استخدمت طريقة النحو والترجمة في مادة الترجمة بلّن يترجم المدرس الكمات من

اللغة الوطنية إلى اللغة العربية أو العكس. واتخدمت طريقة القراءة في مادة المطالعة بعد إعطاء معاني الكلمات الصعبة، بكيث أمر الجرسقراءة المادة عن طريق القراءة الجهرية للتيقن والتأكد من صحة القراءة ثم القراءة الصامتة لفهم النصوص العربية. وللكشف عن مدى فهم الطلاب عما قرأ وا، طلب المدرس بعض الط لاب شرح الموضوع المقروء بينما الأخر يستمع إليه ويناقشه عما يحتاج إلى المناقشة.

واستخدمت طريقة السمعية الشفوية في مادة المحفوظات. فذكر المدرس الكمات

أو العبارات بصوت عال وكررها الط لاب. وهذه العملية تساعدهم التدريب على المفردات أو العبارات إلى أن يتّودون بأنفسه مدون أن يثعرو أنهيوجب عليهم حفظ الكلمات أو العبارات المقصودة. فهذه الطريقة تدربه معلى الممارسة على الأنماط اللغوية حتى يتّودون بها وكياعد هم عند الاتصال باللغة العربية. ليلزم تماما على المدرس استخدامللغة العربية عند شرح المعنى ثم تلفظ الكلمات بيتا فبيتا ، وهو في نفس الوقت قد استخدمت الطريقة المباشرة. وفي تعليم مادة النحو باستخدام الطريقة الإستقرائية ، حيث يبدأ الشرح من الأمثلة ثم يأتي في النهاية بشرح القاعدة النحوية المقصودة. 
فاستنادا من التحليل السابق يمكن القول بأن الطريقة المستخدمة في تعليم

اللغة العربية في المعهد الإسلامي بفونوروغو سلفيا كان أم عصريا هي الطريقة التوليفية حيث أن هذه الطريقة طريقة حديثة الظهور التي تلبي حاجات المدرسين والطلاب في تعلم اللغة العربية حيث أن هذه الطريقة تحاول دمج محاسن الطرق القديمة وطرح مساوئها.

النشاطات اللغوية في تعليم اللغة العربية في المعهد الإسلامي بفونوروغو

وأما الضشاطات اللغوية المعمولة في معهد الرسالة في تعليم اللغة العربية فهي كما

$$
\text { أ) المحادثة اليومية باللغة الرسمية }
$$

من واجبات الطلاب الساطين في معهد الرسالة هو المحادثة باستخدام اللغة

الرسمية للمعهد وهي اللغة العربية واللغة الإنجليزية. وتدور هتين اللغتين كل

الأسبوع. ويسير هذا النظام في كل مكان إما في الغفة الدراسية أو في المطعم أو في

الحمام وكل مكان في حرم المعهد. أما الطلاب الجدد فله مالرخصة حتى نصف السنة

الأولى. يسير هذا النظام على مسؤلية قسم اللغة تحت إثراف مشرف اللغة من 
المدرسين.الهدف من هذا النشاط هو رفع قدرة الطلاب على الكلام باللغة الرسمية

$$
\begin{aligned}
& \text { كل يوم في معاملاتهم مع بني جنسهم. } \\
& \text { ب) الحادثة الصباحية }
\end{aligned}
$$

سارت هذه المحادثة اليومية كل يوم الجمعة بعد صلاة الصبح في الميدان أمام قاعة

الطلاب. يجمع الطلاب في الميدان فصلا فصلا ولكل فصل مدبر ون من السنة السادسق. يجب على كل طالبلمتهدث مع صاحبه مواجها بالموضوع المعين من قسم اللغة. وفي النهاية ، يختار قسم اللغة نفر كن من كل فصل وأمرهما المحادثة أمام أصح ابهم. ؤأما الهدف من هذا النشاط هو رفح قدرة الطلاب على الكلام باللغة الرسمية مح الأصحاب في موضوع معين. ج) إعطاء المفردات ينفذ هذا النشاط كل يوم بعد صلاة العصر ، حيث أن لكل يوم ثلاث مفردات. والمعطيهو مدبرو الحجرات، حيث يعقد الاختبار عن المفردات المعطاة أسبوعيا وذلك في كل يوم الخميس صباحا.

$$
\begin{aligned}
& \text { 14 رزقا رياني، المقابلة 4، في معهد الرسالة، التاريخ } 6 \text { أبريل } 2019
\end{aligned}
$$

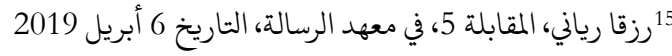

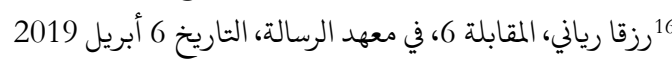

Program Studi Pendidikan Bahasa Arab Universitas Yudharta Pasuruan https://jurnal.yudharta.ac.id/v2/index.php/studi-arab 
166 | Volume 10, Nomor 2, Desember 2019

$$
\text { د) تدريب الخطابة }
$$

يجب على جميع الطلاب في المعهد الرسالة مشاركة تدريب الخطابة مرة في الأسبوع وذلك في يوم الخميس نهارا في الحصة الخامسة إلى الحصة السادسة أي في الساعة الحادية عشرة إلى الساعة الثانية عشر قوالربع. وفي الليل في الساعة السابعة والنصف أي بعد تناول العشاء إلى الساعة التاسعة تماما. يتكون تدريب الخطابة في ثلاث لغات هي اللغة العربية واللغة الإنجليزية واللغة الإندونيسية. أشرف على هذا النشاط قسم التعليم التابع لمنظمة الطلاب. ولهذا النشاط يقسم قسم التعليم الفرق. ولكل فرقة مجموعة من 12 طالبا. نظم رئيس الفرقة الجول لإلقاء الخطابة في الأسبوع الآتي. و دعا رئيس الجلسة من الخطباء واحدا فواحدا. ثم اختار من المستمعين للتقدم لأخذ الإستنباط من خطبة الخطباء. وفي النهاية يصلح الملاحظون الكلمات الخاطئة وأعلن النتيجة لمن حسنت خطابق 17 أما الهدف من هذا النشاط رفع قدرة الطلاب على إستماع الخطبة باللغة العربيةحتيستطيعون أخذ الإستنباط والمغزى مما سموا. ه) التجسس والمحكمة اللغوية يهدف هذا النشاط إلى التأكد في أن الطلاب يتحدثن باللغة الرسمية كل يوم في كل مكان. لكل طالب وظيفة كتابة اسم المخ الف الذييتحدث باللغة غير رسمية في ورقة

$$
\text { 17 رزقا رياني، المقابلة 7، في معهد الرسالة، التاريخ } 6 \text { أبريل } 2019
$$

Program Studi Pendidikan Bahasa Arab Universitas Yudharta Pasuruan https://jurnal.yudharta.ac.id/v2/index.php/studi-arab 
معينة من قسم اللغة وتجمع الورقة في صندوق التجسس. ولسير هذه النشاط يقسم

$$
\text { قسم اللغة فرق التجسس من كل فصل.18 }
$$

وبعد إنتهاء التعلم الليلي ،أعلن قسم اللغة، أسماء المخلفينللحضور إلى ديوان قسم اللغة لأداء التحكيم. التحكيم هو نشاط لإصلاح الكلام المخظئ . وهذا نشاط مهم

$$
\text { و في معهد الرسالة لوجوب التحدث باللغة الرسمية. }
$$

يهدف هذا النشاط إلى تنمية مهارة الكلام. يؤدي كل شهر، و تكون المسابقة

بين مساكن الطلاب ـ قسم قسم اللغة الموضوع، وقبل التقدم وجب على

الطلاب القدم إلى مشرف اللغة لإصلاح محادثته عند مسابقةالتمثيل

$$
\text { المسرحي. }
$$

$$
\text { 2) - 2 }
$$

في هذا النشاط يجب على الطلابكتابة مقالة عن حكاية أو قصة معينة باللغة

العربيةثم جمعها إلى مشرف اللغة ل لإِصلاح. في المسابقة يجب على

الطالبالمشارك أن يحكي حكاية دون النظر إلى المقالة وبلستعمال الحركة. وهذا

$$
\text { النشاط يهدف إلى تنمية مهارة الكلام والكتابة. }
$$

$$
\text { 18 رزقا رياني، المقابلة 8، في معهد الرسالة، التاريخ } 6 \text { أبريل } 2019
$$

Program Studi Pendidikan Bahasa Arab Universitas Yudharta Pasuruan https://jurnal.yudharta.ac.id/v2/index.php/studi-arab 
168 | Volume 10, Nomor 2, Desember 2019

3) - (3) - (3سابقة النقاش

تقسم لجنة المسابقة الطلاب إلى أربع فرق ، فرقلن للكبار وفرقتان للصغار.

والموضوع المبحوث معين من قيل لجنة المسابقة ولكن المقالة من نفس أعضاء

المسابقة. فني المسابقة يجب على كل أعضاء المسابقة ممارسة اللغة العربية لإبداء

آراءهم عن الموضوع المطروح. المدف من هذه المسابقة هو تنمية مهارة الكلام

لدى الطلاب.

أما النشاطات اللغوية المنفذة فهي كما يلي :

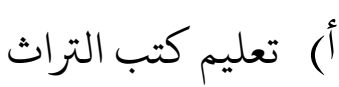

يهدف هذا النشاط تنمية إلى قدرة الإستماع والقراءة باللغة العربية. وهذا النشاط

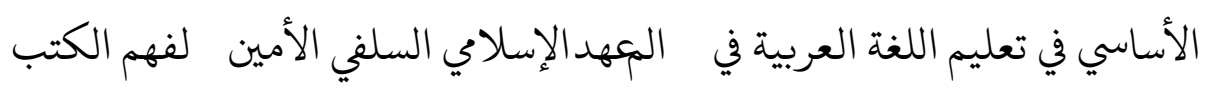

العربية و الكتب الإسلامية. ولأهمية هذا النشاط، فإنه ينفذ كل يوم خصوصا بعد

صلاة العشاءو بعد صلاة الصبح عن طريق التقديم ( sorogan) أو التوقيت

(peton/ bandongan)

ب) المحافظة والمشاورة

نفذ هذا النشاطلتنمية مهارة الإستماع والقراءة باللغة العربية. المحافظة هي وجوب

الحفظ النظم لمحتوى كتاب معين. والمشاورة هي تدريب القراءة للكتب العربية. 


\section{ج) بحث المسائل}

يسير هذا النشاط شهريا. وأما المدفعن هذا النشاط هو تنمية قدرة الطلاب في قراءة

الكتب والمراجع أو المصادر العربية من خلال البحث عن إجابة المشكلة

$$
\text { الإجتماعية. }
$$

فمن البيان الموجز السابق ذكره يمكن إجراء المقارنة بين المعهدين العصري

والسلفي في الأنشطة اللغوية في تعليم اللغةالعربية على نحو الجدول التالي :

\begin{tabular}{|c|c|c|c|}
\hline ب ب & i & النشاطات & الرقم \\
\hline & & محادثة اليومية باللغة الرسمية & 1 \\
\hline & & محادثة الصباحية & 2 \\
\hline & & تدريب الخطابة & 3 \\
\hline & & التسميع & 4 \\
\hline & & التجسس والمحكمة اللغوية & 5 \\
\hline & & المسابقات اللغوية & 6 \\
\hline & & تعليم كتب التراث & 7 \\
\hline & & المحافظة والمشاورة & 8 \\
\hline & & بحث & 9 \\
\hline
\end{tabular}


170 | Volume 10, Nomor 2, Desember 2019

$$
\text { ب أ :معهد الرسالة }
$$

تتنوع النشاطات اللغوية في المعهد العصري إلى نوعين هي الضشاطات اللغوية داخل الغرفة الدراسية، منها: وجوب الكلام والتحدث باللغة العربية ، والتكلم أوحفظ مادة اللغة العربية، والضشاطات اللغوية خارج الغرفة الدراسية،منها: المحادثة الصباحية، وتدريب الخطابة، والتسميع والتجسس والمحكمة والمسابقات اللغوية. أما النشاطات اللغوية المنفذة في المعهد السلفي فتدور حول تعميق الطلاب في فهم النصوص العربية وكتب التراث الإسلامية، إضافة إلى ذلك فإن المعهد السلفي له ضوابط ومعايير خاصة في معالجة القواعد النحوية وحفظها حفظا تاما وقيقا من خلال تطبيق النشاطات اللغوية.

إن مواد تعليم اللغة العربية في المعهد تتكون من المواد: النحو والصرف والإنشاء والمطالعة والإملاء والخط والمحفوظات. أما في المعهد السلفي فتتكون من الصرف والنحو والجوومية و العمريطي والألفية، و أنها نظمت منطقيا على أساسي التنظيم المترابط ـ أما الطريقة المستخدمة في تعليم اللغة العربية في المعهد العصري هي طريقة قواعد والترجة والطريقة المباشرة والطريقة السمعية الشفوية و طريقة القراءة والطريقة الإستقرائية. أما الطريقة المستخدمة في تعليم اللغة العربية في المعهد السلفي هي طريقة النحو والترجة 
والطريقة القياسية ، و بذلك طبق المعهد الإسلامي الطريقة التوليفية في تعليم اللغة العربية، وهي الطريقة التي تلبي حاجات المدرسين والطلاب حيث أنها تحاول أخذ محاسن الطرق القديمة وطرح مساوئها، بينما النشاطات اللغوية المنفذة في المعهد الإسلاي تتنوع تنوعا ملائما حسب تنوع أهداف تعليم اللغة العربية في كلا المعهدين العصري والسلفي، وأنها طبقت داخل الغرفة الدراسية وخارجها.

المراجع

بحر الدين،أوريل .تطوير منهج تعليم اللغة العربية وتطبيقه على مهارة الكتابة ، مالانج: مطبعة الجامعة الإسلامية الحكومية، 2010.

الديلمي، طه علي حسين ـ اللغة العربية مناهجها وطرائق تدريسها ، الأردان: دار الشروق، 2005

الخول، محمد علي.أساليب تدريس اللغة العربية. الأدن:دار الفلاح للنشر والتوزيع، 2000. رسلان، مصطفى.تعليم اللغة العربية. القاهر: دار الثقافة للنشر والتوزيح. 2005.

الشافي، ابراهيم محمد، و اخرون، المنهج المدرسي من منظور جديد، القاهرة: درا الفللاح، 2009

شهداء. مناهج تعليم اللغة العربية في المدارس الابتدائية باندونيسيا (دراسة تحليلة تقويمية). بحث مقدم لنيل درجة الدكتور اه في التربية (المناهج وطرق التدريس)،

$$
\text { غير منشور، الخرطوم: } 2004
$$

Program Studi Pendidikan Bahasa Arab Universitas Yudharta Pasuruan https://jurnal.yudharta.ac.id/v2/index.php/studi-arab 


\section{صالح الدين، أحمد. تعليم اللغة العربية في المهد السلفي والمعهد العصري (دراسة مقارنة وتقويمية في تعليم اللغة العربية بين معهد ليربويو السلفي وبقديري والأمين}

$$
\text { العصري بسومنب)، بحث مقدم لنيل درجة الماجستير في التربية، } 2007
$$

طعيمه، رشدى أحمد .تعليم اللغة العربية لغير الناطقين بها مناهجه وأساليبه. رياط:

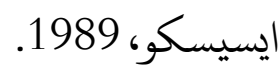

منير، محمد. تعليم اللغة العربية في المعهد العصري والمعهد السلفي، مالانج: الجامعة

$$
\text { الإسلامية الحكومية مالانج، غير منشور، رسالة دكتوراه، } 2014 .
$$

Arikunto, Suharsimi. Prosedur Penelitian Suatu Pendekatan Praktek. Jakarta: PT. Rineka Cipta, 2002.

Basrowi dan Suwandi. Memahami Penelitian Kualitatif. Jakarta: Rineka Cipta, 2008.

Hamid, Abdul, Uril Bahruddin, Bisri Musthofa. Pembelajaran Bahasa Arab Pendekatan, Metode, Strategi, Materi dan Media. Malang: UIN Malang Press, 2008.

Sugiyono. Metode Penelitian Kuantitatif Kualitatif R\&D. Bandung: Alfabeta, 2011. 